\title{
PITFALLS IN GRAF'S METHOD FOR EARLY ULTRASOUND SCREENING OF HIP DISPLASIA
}

\author{
Raykov D. \\ Department of orthopedics and traumatology, Medical university - Varna
}

Reviewed by: Prof. D. Boshnakov

\begin{abstract}
The ultrasound screening of baby's hip is one of the most valuable method for early diagnosis of congenital or developmental hip dysplasia. Graf's method is a popular worldwide, having thousands of followers. Every observer must know as the normal ultrasound anatomy, as the hip pathology images. He also must be aware of possible errors, coming from the improper use of devices (console softwear options and transducers) and some image distortions, resulted by the "tilt" effect. Thus the method will avoid possibility to give wrong diagnosis and to mislead as the observer as all other specialist, who care for the baby's health.
\end{abstract}

Key words: DDH, Graf's method, pitfalls in ultrasound screening of baby's hip

\section{INTRODUCTION}

Ultrasound (US) screening of baby's hip $(1,2)$, established by professor Graf from Austria has more than 25 years history (4). There are thousands of followers of this method. The popularity and positive results are getting more and more popular all around the world. It is pointed out as one of the biggest and most important application of ultrasound in medicine. Some authors like Niethard F. Aachen, Germany dare to describe it as the most effective method for prevention in orthopedics (2).

The current research has the aim to present some possible points of mistakes that may alter the proper final diagnoses and thus to mislead all medics, who care for baby's health $(5,6)$.

\section{MATERIAL AND METHODS}

The following study encounters 4 chapters, analyzing the equipment, some practical advices and tips, that point out fields of possible mistakes.

\section{Devices that should be taken in use.}

1. Console and transducers. The compulsory part of the device configuration is the presence of linear transducer (8) with different frequency -5-7,5-9-12 Mhz (Fig.1). Increasing the frequencies the observer gets higher resolution and quality of the image, but the penetration becomes worse. This fact should be taken in mind in

\footnotetext{
Address for correspondence:

D. Raykov, Dept. of Orthopaedics and Traumatology

Medical University - Varna

55 "Drinov" Str., 9001, Varna, Bulgaria

e-mail: raikovortho@dir.bg
}

screening babies over 4 months of age, who have bigger thickness of subcutaneous fat and it could disturb the clear image of the deep lower limp of the hip. According the original method the optimal frequency of the transducer should be 7,5 MHz.

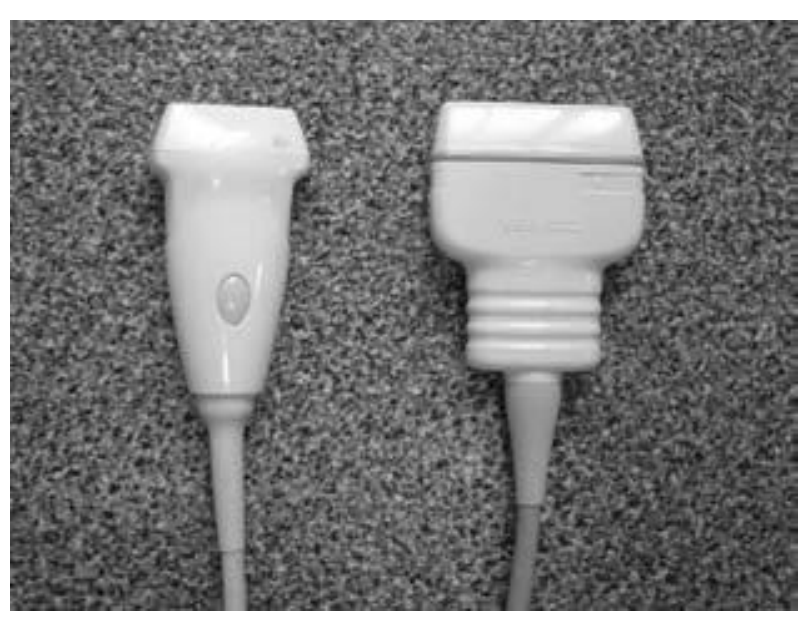

Fig. 1. Linear transducers with different head dimensions

2. Foot switch. If the device is not accomplished with such element, then the observation could become longer and more difficult. Thus the baby becomes nervous; somebody else should press the freeze button on the device (usually the mother of the child).

3. The documentation should be done by either a termoprinter or a standard computer printer (10). The observer must do two pictures of each hip, one of them is for the babies history and the other is for the doctor's archive. If the pictures are made on termopaper, they should always be kept in dark folders and to be 
preserved from direct light. Otherwise they will fade and will loose their image qualities. This will disturb eventual need of comparison with further US pictures or consultation with expert. From practical point of view it is useful to prepare MS office (Word) files capturing the images and finally print them by a normal printer and to save them in the doctor's computer.
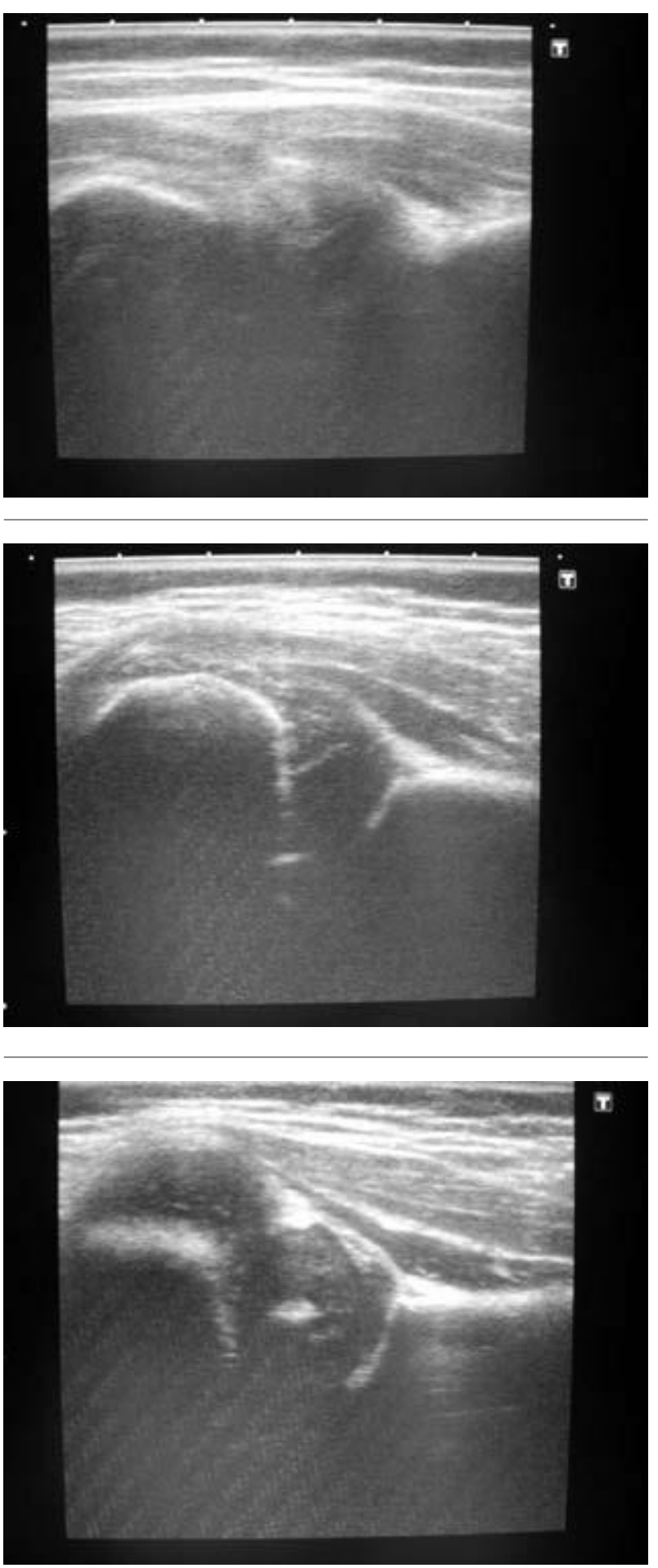

Fig. 2-A,B,C. "Cinema" function gives possibility for digital adjustment of the most appropriate picture moving it backward and forward.
4. Internal device software for angle measuring. This process must be done by the observer manually.

5. Cinema function of the console - a very good and profitable option, that makes possible choosing the best image while moving it forward and backwards until acquiring the best Graf's image (Fig. 2-A,B,C).

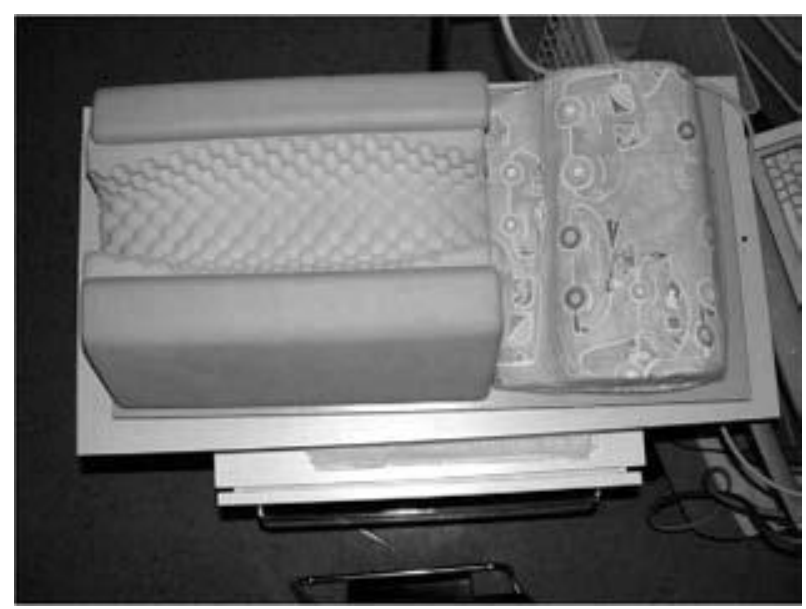

Fig. 3-A. Cradle for lateral positioning of the baby

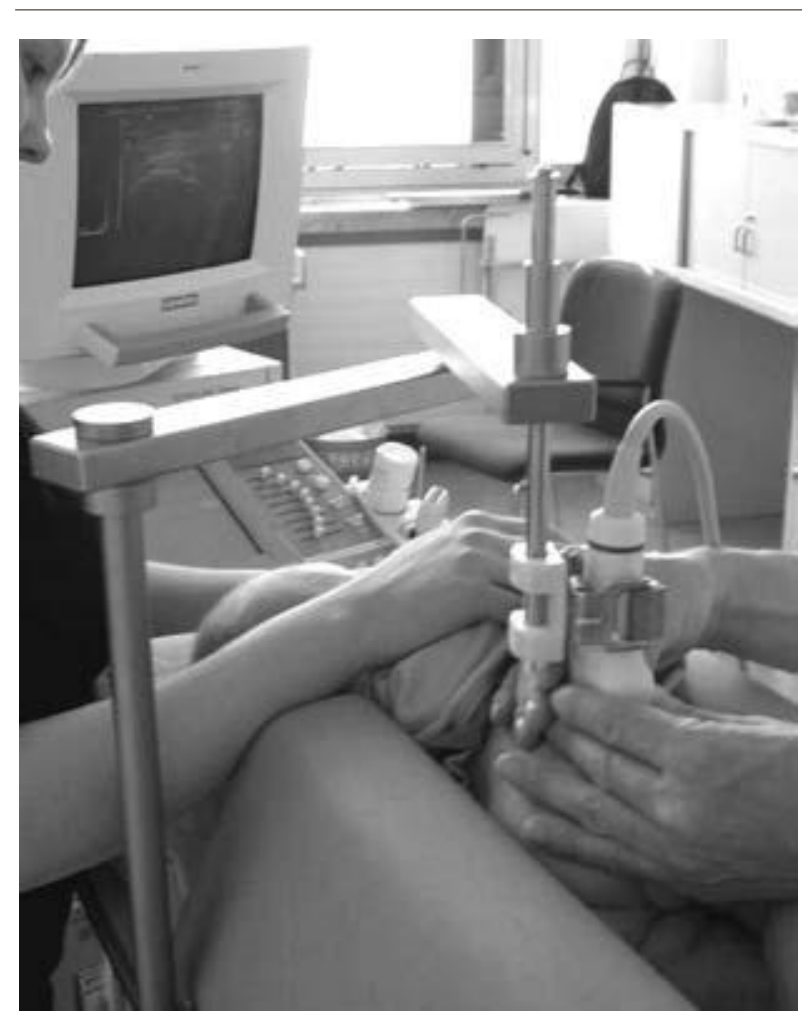

Fig. 3-B. Targeting device for strict positioning of the transducer to the body.

6. Cradle and targeting systems (9) for best position of the body and maximum exclusion of possible image deviation because of misposition (Fig. 3-A,B).

\section{Possible mistakes in device choice are as follows:}


1. Use of convex transducers is a mistake (3). Even being more popular in current medical practice (abdominal sonography) it should not be used in ultrasound hip sonograpfy (Fig. 4). Specific distortion and deviations

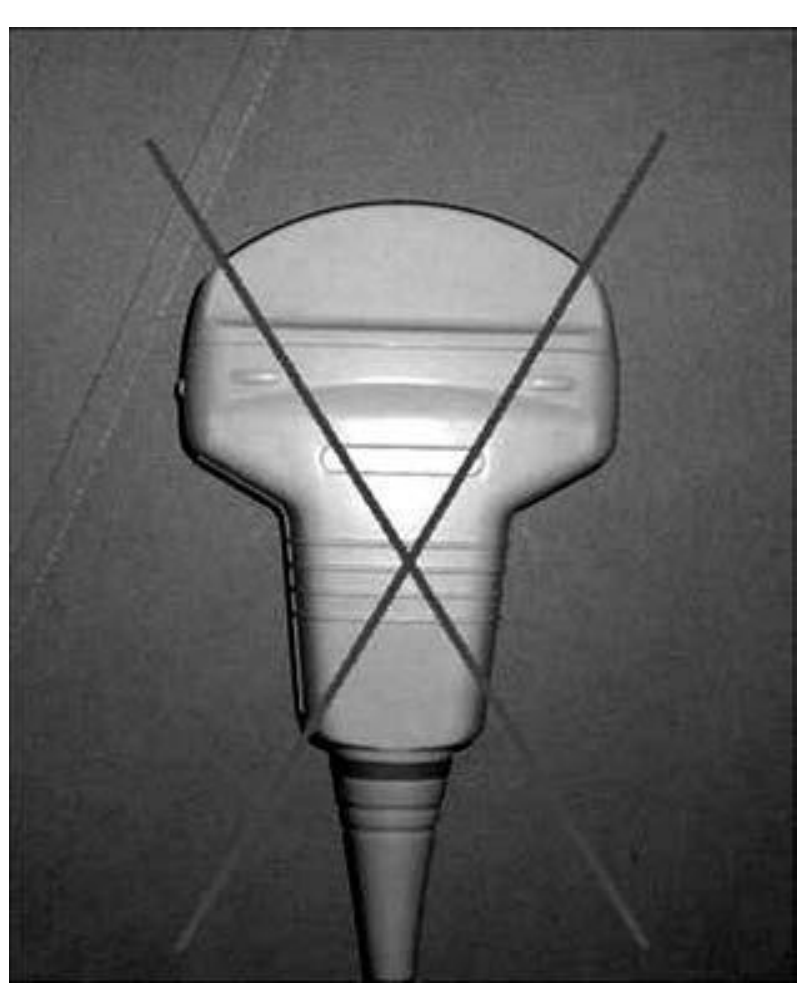

Fig. 4. Convex transducer gives distortion of the linear hip image and must not be used.

will destroy the linear image according to Graff's method and will give improper image.

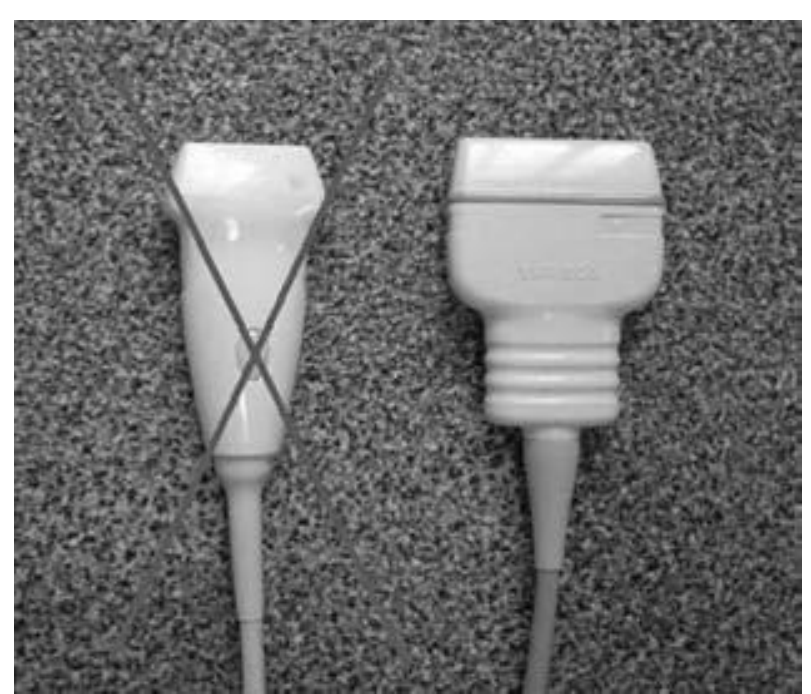

Fig. 5. Linear transducers, that head is les than $4 \mathrm{~cm}$ is not appropriate, because cannot catch the Graf's $\mathbf{L P L}$ triad (lower limp, plane, labrum).

2. Use of linear transducers, smaller than 40 millimeters. These transducers are popular in endocrinology, hematology, rheumatology, mamology for observation of small body parts. The small head of the transducer does not allow getting the full and compulsory triad LPL (presence of lower limp, plane, labrum) that are compulsory elements for the proper diagnosis (Fig. 5).

3. Software for automatical measurement of $\alpha$ and $\beta$ angles could make mistakes and mislead the observer. The measurement should always be done manually.

\section{Practical advices}

1. The baby must be tested manually (Fig. 6) after accomplishing the US procedure. In this way it will stay relaxed and calm while doing ultra sound. Otherwise it becomes nervous and stressed.

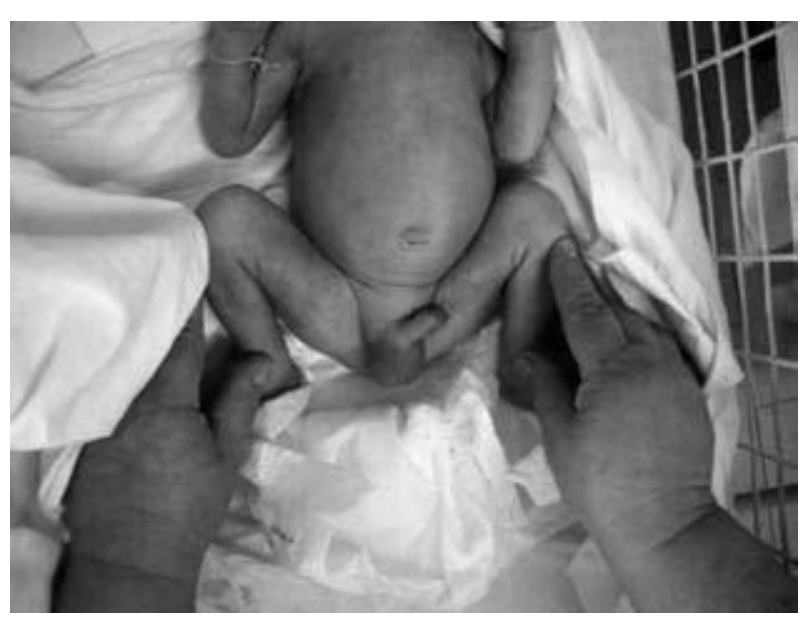

Fig. 6. Manual testing of the baby must follow the US screening to keep the baby calm.

2. The baby should be undressed in another room for preparation in order to keep its calm mood.

3. If the cradle and the targeting systems are missing, the baby must be kept in lateral position (Fig. 7) that is closest to the Graf's one and provides possibility for slight internal rotation to intrude the proximal femur in the image.

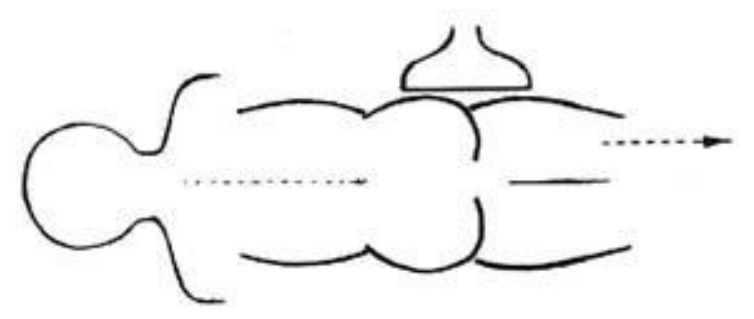

Fig. 7. Lateral position of the body and transducer to its frontal plane.

\section{Tilt effect}

It is distortion of the ultrasound image because of improper positioning of the transducer towards the plane and axis of the body, followed by improper interpretation (7). It is of a great value for the observer to know the possible tilting effects and their typical pictures to ignore them, while giving the diagnosis. 


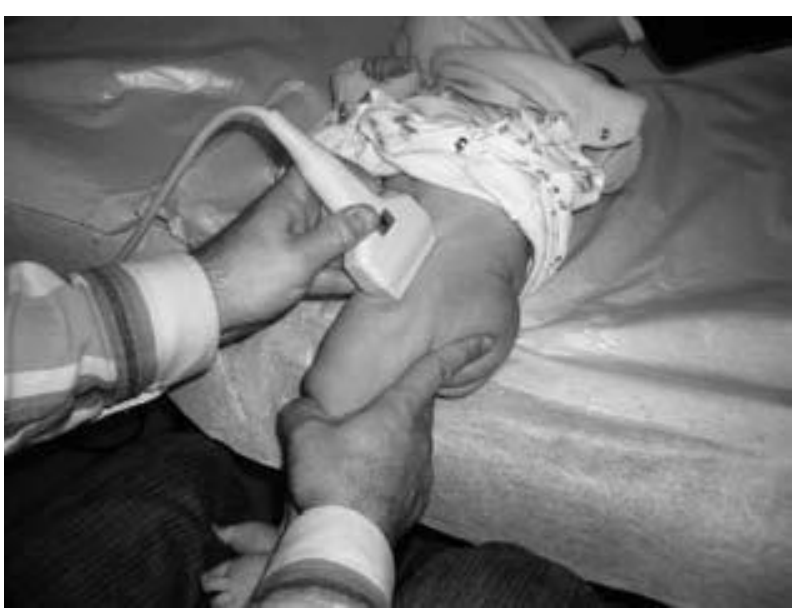

Fig. 8-A. Anterior posterior tilt - the transducer is tilted from anterior up to posterior down direction.

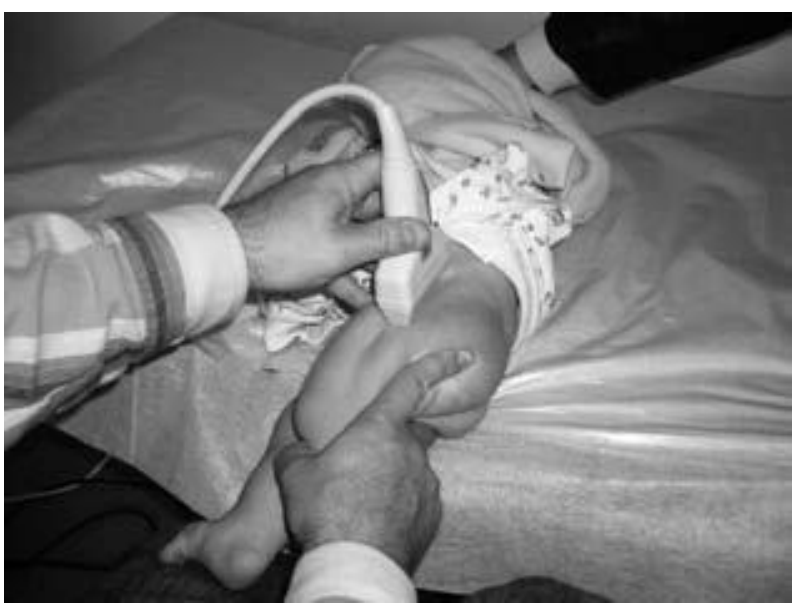

Fig. 8-B. Normal transducer position in the frontal plane.

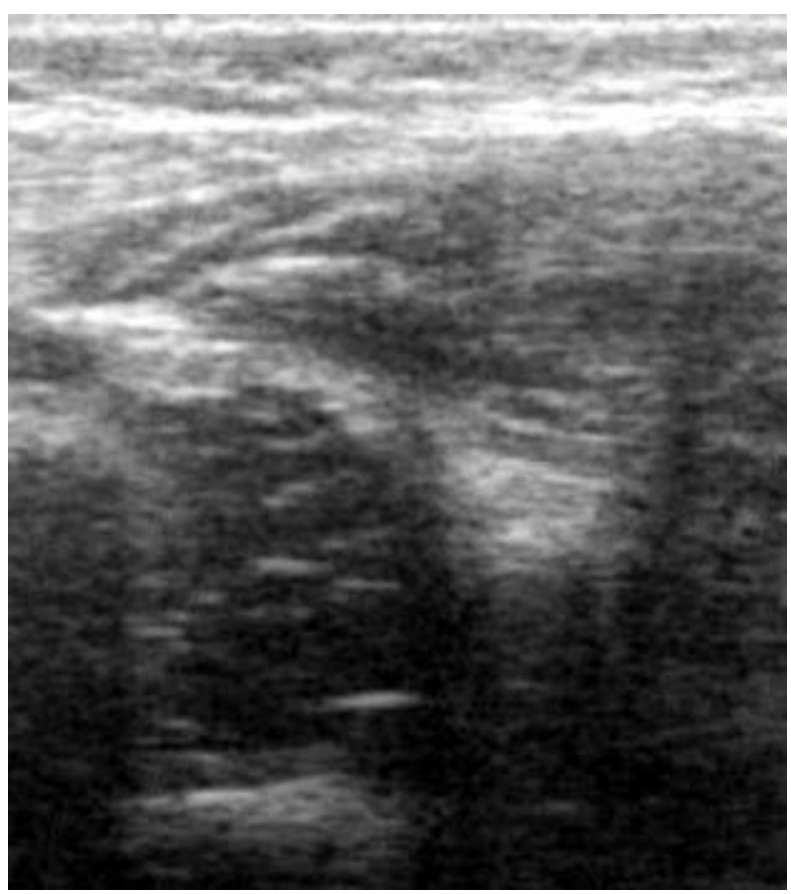

Fig. 8-C. US in anterior-posterior tilt - blurred ileum and impossible $\alpha$ measuring.

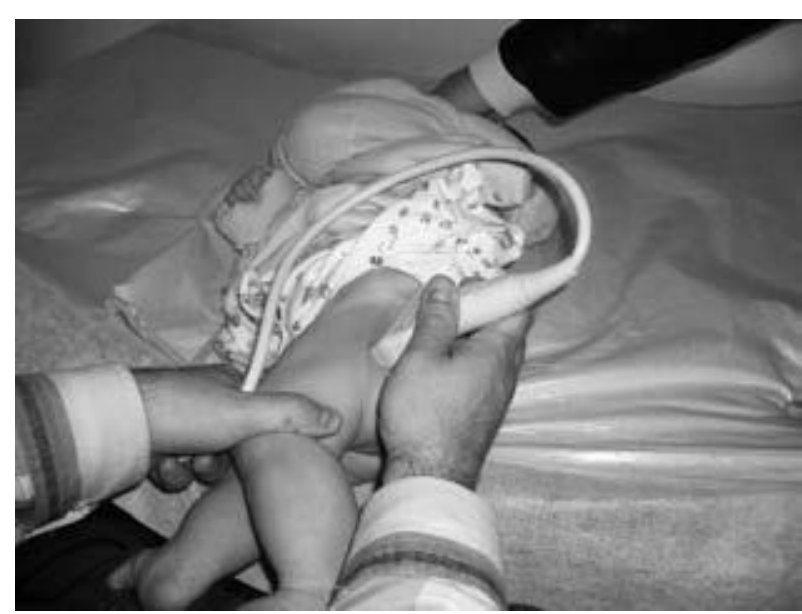

Fig. 9-A. Posterior anterior tilt: the transducer is tilted from posterior up to anterior down direction.

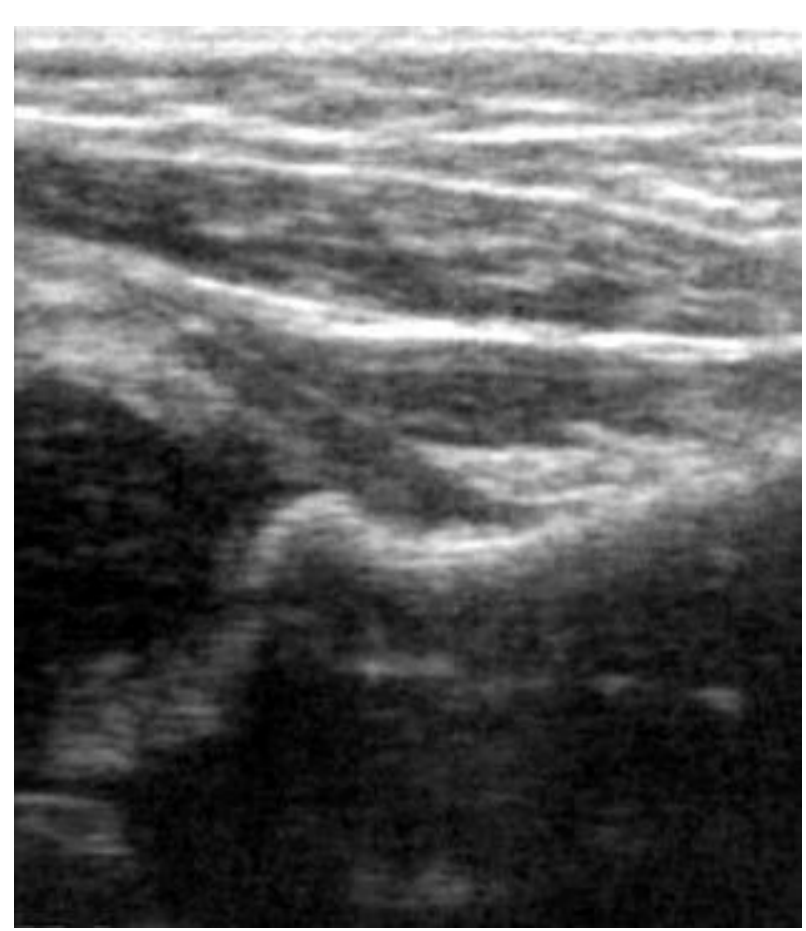

Fig. 9-C. US in posterior - anterior tilt-curved ileum and rim image like bird's beak; possible mistake in á measuring.

\section{Antero-posterior tilt}

The transducer is tilted anterior to posterior in spite of being parallel to the body axis.

The image is very close to the normal, but the acetabular limbus is very hard to be defined (the whole ilium is blured), that could cause a mistake in angle measurement Fig. 8-A (wrong), Fig. 8-B (right), with US pictures - Fig. 8-C (wrong), Fig. 8-D (right).

\section{Posterior- anterior tilt}

The transducer is tilled from posterior to anterior direction. This leads to a typical acetabular rim image like bird's beak. It disturbs the angle measurement: Fig. 9-A (wrong), Fig. 8-B (right), with US pictures - Fig. 9-C (wrong), Fig. 8-D (right).

\section{Cranio-caudal tilt}




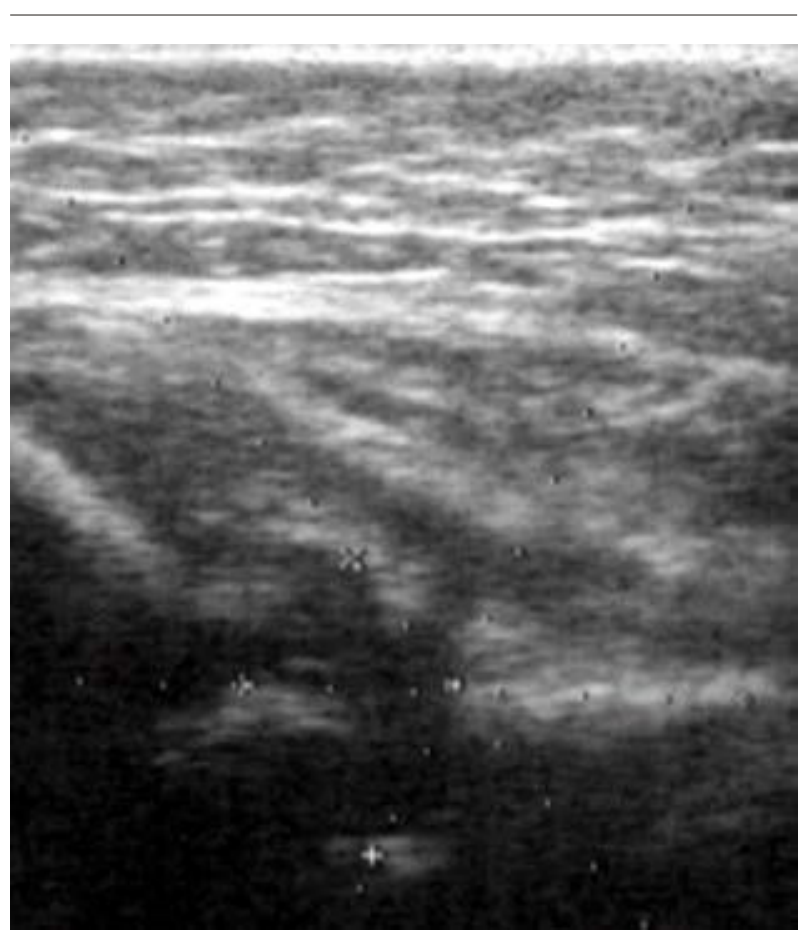

Fig. 8-D. Normal US picture with LPL triad.

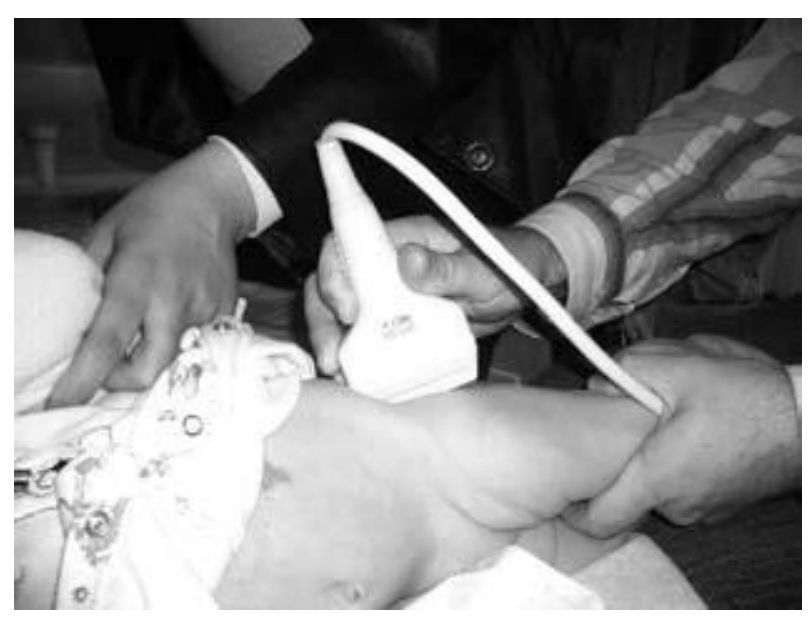

Fig. 10-A. Cranio-caudal tilt. The transducer is in the frontal plane, but is tilted from cranial to caudal direction.

The tranducer is in frontal plane of the body, but is tilted caudally.

It looses the image of the lower limp and makes the measurement of $\alpha$ angle improper. Fig. 10-A (wrong), Fig. 8-B (right), with US pictures - Fig. 10-C (wrong), Fig. 8-D (right). The drawings Fig. 10-E (wrong) and Fig. 10-F (right) prove the distortion.

\section{Caudo-cranial tilt}

This is the most common mistake that makes improper measurement of the acetabular roof and of false image for decentrated or dislocated femoral head. This could bring the following mistakes in $\alpha$ angle. Fig. 11-A (wrong), Fig. 8-B (right), with US pictures - Fig. 11-C (wrong), Fig. 8-D (right). The drawings Fig. 11-E (wrong) and Fig. 10-F (right) prove the distortion.

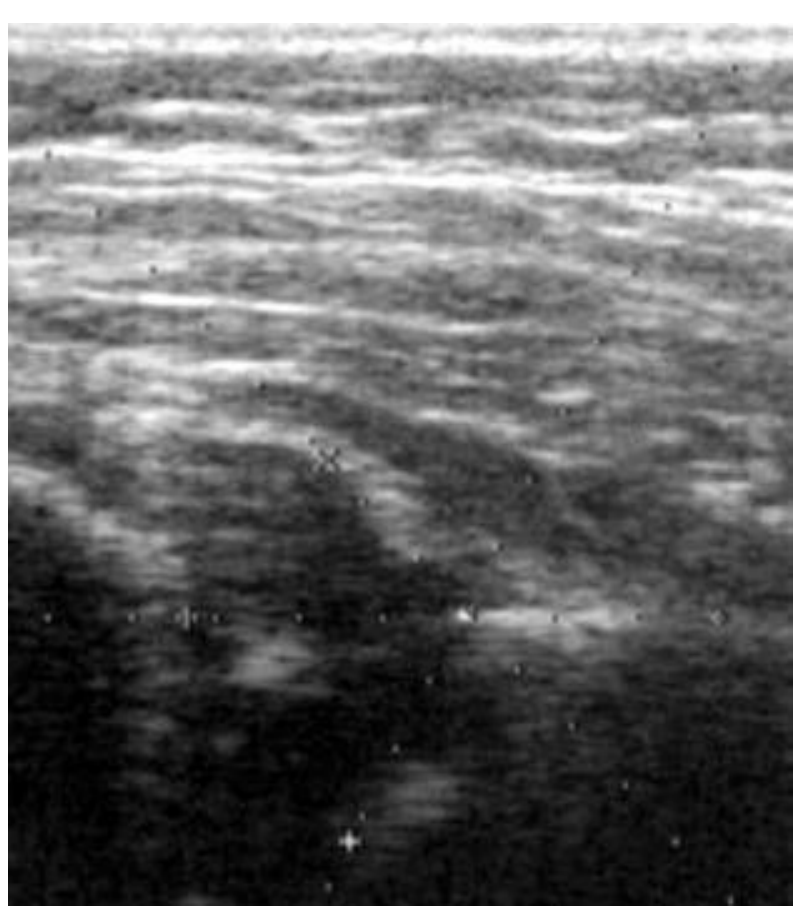

Fig. 10-C. The lower limp is miss positioned and makes the measurement of á angle improper.

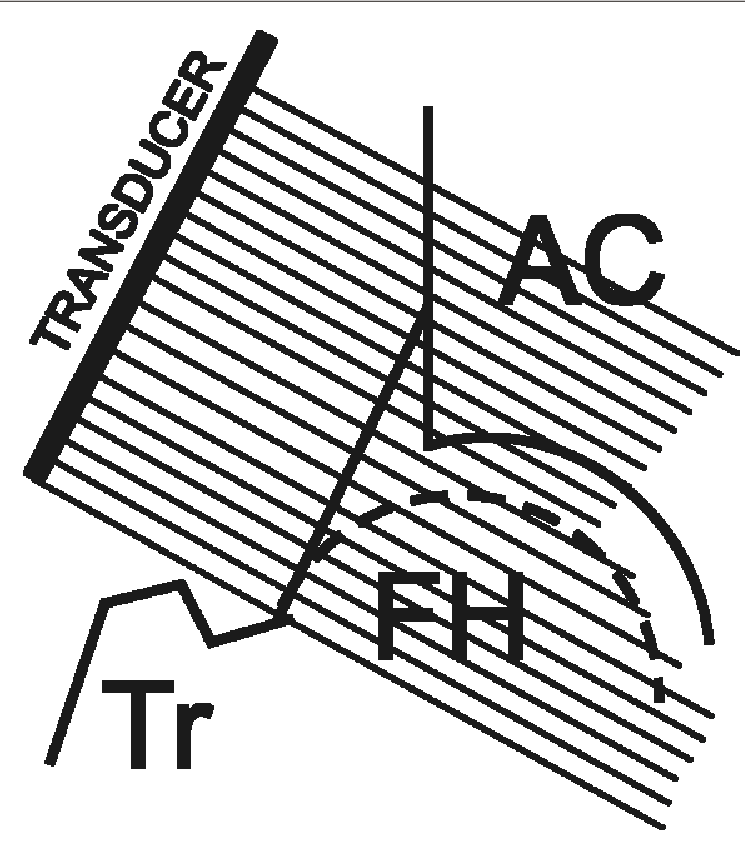

Fig. 10-E. Drawing, explaining the distortion.

- 10 degrees of caudo-cranial decreases $\alpha$ angle with 10 degrees;

- 20 degrees of transducer tilt decreases the real $\alpha$ angle with 20 degrees.

\section{CONCLUSIONS}

Absence of standard accomplishment, the proper choice for ultrasound device and periphery are common problems for most of the orthopedic clinics and offices. 


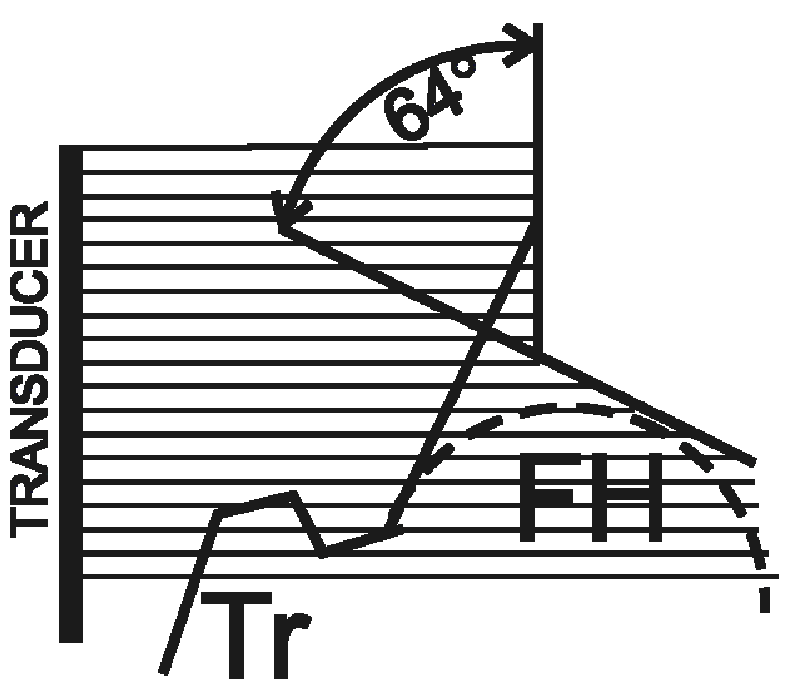

Fig. 10-F. Drawing - linear penetration of the US rays without distortion, when the transducer is properly positioned.

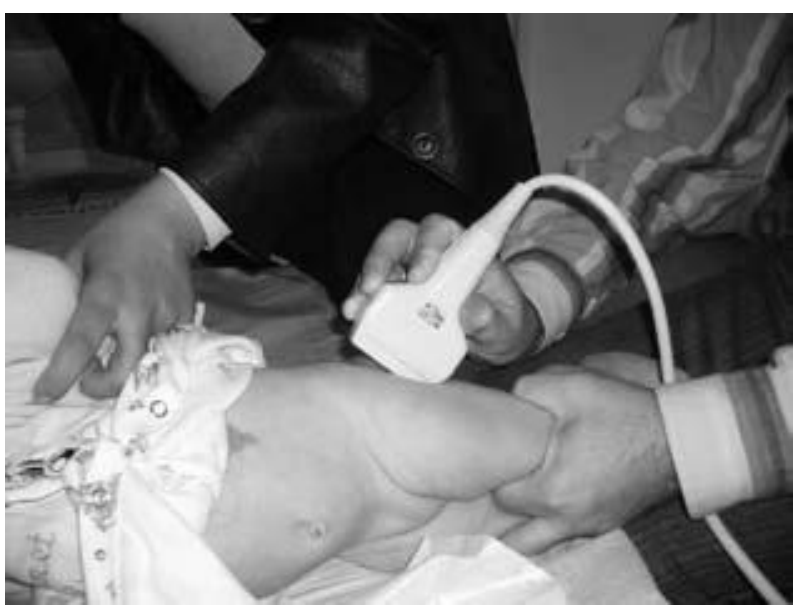

Fig. 11-A. Caudo cranial tilt. The transducer is in the frontal plane, but tilted from caudal to cranial direction.

Being aware of all possible points of mistakes, the observer must diminish to maximum the mistakes that alter the diagnosis.

\section{BIBLIOGRAPHY}

1. Benner G C: Screening for congenital dislocation of the hip. J Bone Joint Surgery (Br) (1992) 74-B: 643-644.

2. Bond Ch D, Henaricus W L, DellaMagione E D: Prospective Evaluation of newborn softtissue hip " Clics) with ultrasound. J Pdiatr Orthop 17(1997) 199-200.

3. Graf R: Hip sonography - How reliable? Sector scanning versus Liner scanning? Dynamnic versus static examination? Clinical Orthopaedics 281; 18-21( August 1992).

4. Graf R: Neonatal sonographic screening for DDH. BMUS Bulletin ay 1993; 22-27.

5. Graf R, Tschauner Chr, Klapsch W: Progress in prevention of late developmental dislocation of the

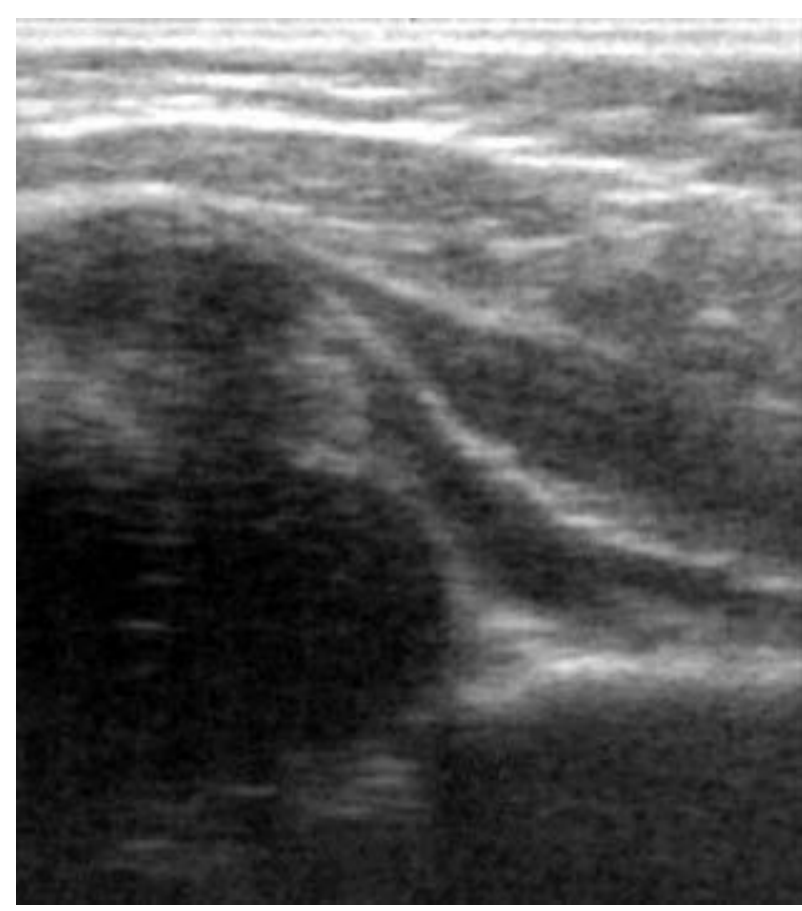

Fig. 11-C. The acetabulum is false shallow; the hips is miss determined as dysplastic.

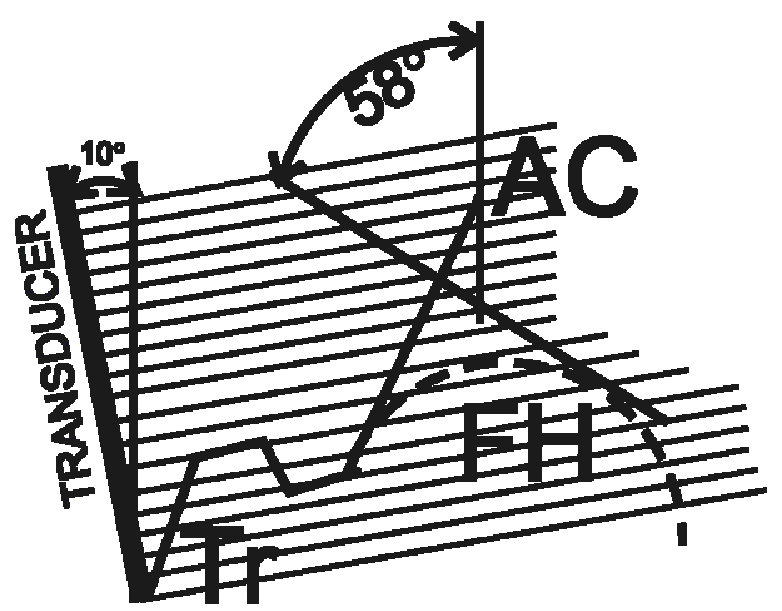

Fig. 11-E. Drawing, explaining the linear distortion.

hip by sonographyc newborn hip"screening": Results of comparative follow up study. J Pediatr Orthop (Part B) 2: 115 - 121.

6. Graf R, Tschauner Chr: Ultrasound screening in the neonatal period. Bailliere's clinical orthopaedics Vol 1,No 1, August 1996, 117-133.

7. Graf R: Hip sonography Diagnosis and management of infant hip dysplasia, Second edition, Springer Verlag Berlin Heidelberg 2006; 76-77.

8. Graf R: Hip sonography Diagnosis and management of infant hip dysplasia, Second edition, Springer Verlag Berlin Heidelberg 2006; 64-69.

9. Graf R: Hip sonography Diagnosis and management of infant hip dysplasia, Second edition, Springer Verlag Berlin Heidelberg 2006; 77.

10. Graf R: Hip sonography Diagnosis and management of infant hip dysplasia, Second edition, Springer Verlag Berlin Heidelberg 2006; 82. 